\title{
The Research of Three Regions Acquisition and Analysis System of Pulse Based on Flexible Sensor
}

\author{
Xue Shilei ${ }^{1, *}$, Hao Zhao ${ }^{1}$, An Ying ${ }^{1}$, and Huang Kun ${ }^{2}$ \\ ${ }^{1}$ China flight test and Research Institute, 710089 Xi'an \\ ${ }^{2}$ School of precision instrument and Optoelectronic Engineering, Tianjin University, 300072 Tianjin
}

\begin{abstract}
The objectification of pulse diagnosis is very important to the development and inheritance of TCM, the first step is how to collect more abundant and comprehensive pulse information quickly, reduce the threshold of users for using pulse diagnosis equipment. The existing pulse diagnosis equipment has some limitations, such as single acquisition site, complex compression form and serious dependence on professionals for correcting-pulse position selection. Therefore, a three-pulse diagnosis system based on flexible sensor is designed, which uses a new type of flexible sensor as the data acquisition port, combined with upper computer software and lower computer software to achieve goals of intelligent decompression and data acquisition from Cun, Guan, Chi. The equipment not only greatly reduces the difficulty for users to find correct pulse position identification, but also collect non-destructive pulse information, which provides a new acquisition mode for the pulse diagnosis instrument.
\end{abstract}

\section{Introduction}

Pulse diagnosis is to monitor or diagnose the operating state of the whole body and local organs by observing the subtle changes in characteristics of the morphological carrier, such as pulsation and pulse condition. Generally speaking, blood is pumped from the heart and flows through various organs of the body, which carrying various information about human physiology and pathology. Traditional Chinese medicine doctors apply different finger force using abdomen of the fingers to press the radial artery to obtain the patient's information. Existing pulse acquisition equipment using the same way to detect pulse signal ${ }^{[1]}$.

However, most of the existing acquisition equipment adopts piezoresistive sensors, which are built-in Wheatstone Bridge and directly in contact with the human skin. On the one hand, prolonged pressing on the skin can cause injury. On the other hand, the built-in Wheatstone Bridge is prone to damage. According to statistics, $62 \%{ }^{[2-4]}$ of the existing pulse acquisition equipment is damaged due to sensor damage. In addition, the existing pulse acquisition equipment often requires users to find the pulse location accurately, which largely limits the development of pulse diagnosis instrument and the process of objectification of traditional Chinese medicine.

Therefore, in combination with the principle of pulse diagnosis in Chinese medicine, this paper uses aerodynamics and wave propagation theories to build the optimal pulse-taking pressure model and signal transduction model. On this basis, three regions acquisition and analysis system of pulse based on flexible sensor are designed, which can not only reduce the difficulty of users to a great extent ${ }^{[5]}$, but also collect more comprehensive pulse information. In addition, it provides more accurate information support for precise pulse replay technology and telemedicine.

\section{Figures and tables}

Three regions acquisition and analysis system of pulse based on flexible sensor mainly consists of flexible sensor collector, pressure control module, signal preprocessing module, ARM microprocessor module, serial communication module and upper computer software. The overall design block diagram is shown in Figure 1.

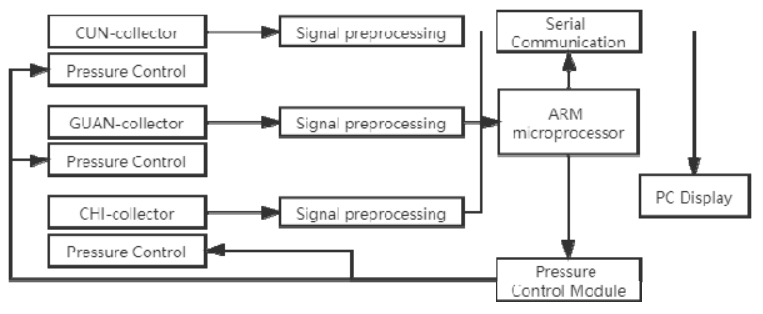

Fig. 1. Structure diagram of three-part pulse instrument

\subsection{Design of flexible sensor collector}

The flexible sensor collector is composed of a flexible silicone air bag with a customized thickness of $0.8 \mathrm{~mm}$, an airway, a P-300C pressure sensor and a wrist strap

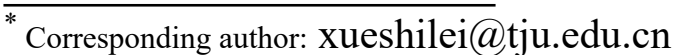


ring. The contact surface between the flexible silicone air bag and the wrist is designed as arc, which can better fit the skin, and the retractable folds can ensure that the extrusion pressure is vertical and downward. The upper part is a through-the-air duct. The width of wristband ring is only $2 \mathrm{~cm}$, and the total width of three wristband rings is $6 \mathrm{~cm}$, which meets the requirements of pulse diagnosis of traditional Chinese medicine. The physical figure of the designed acquisition end is shown in Figure 2.

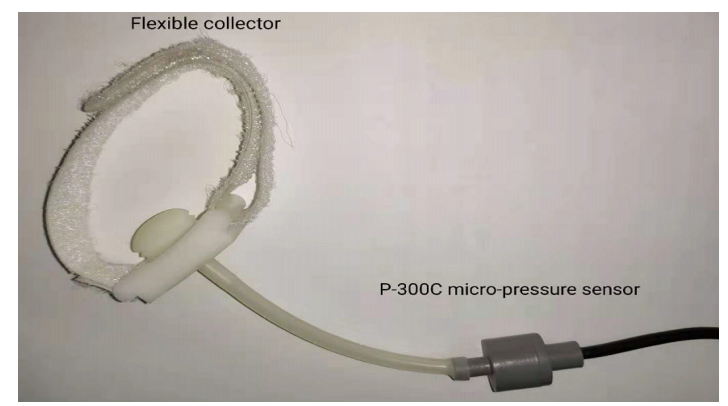

Fig. 2. Flexible sensor acquisition module physical map

The sensitivity of P-300C micro pressure sensor is $0.2 \mathrm{mv} / \mathrm{mmhg}$. It is connected with the air bag through BPT catheter, and the pulse signal is obtained by detecting the intrinsic pressure change in the air bag. The pressure limit of the sensor is $300 \mathrm{mmhg}$, which is much larger than the range of pressure value changes in the airbag. In addition, the sensor has a built-in temperature compensation circuit to ensure the consistency of the collected signal, and its high sensitivity ensures that the transducer can detect the gas static pressure and the gas pulsating pressure, which meets the requirements of the system.

\section{2 signal pre-processing module}

The three processing signals are independent and do not affect each other. The output signal of the flexible pressure sensor is preliminarily processed by the signal pre-processing module, which is based on the AD620 and amplifies the millivolt signal to the ADC's acquisition range. The reference potential of AD620 is $2.5 \mathrm{~V}$, which can avoid negative pressure signal loss. Considering that the main frequency components of human pulse waves are concentrated between $0.08 \mathrm{~Hz}-$ $20 \mathrm{~Hz}$, pre-treatment signals can extract static and dynamic signals (Pulse signal) through filter circuits. The signal pre-processing process is shown in Figure 3.

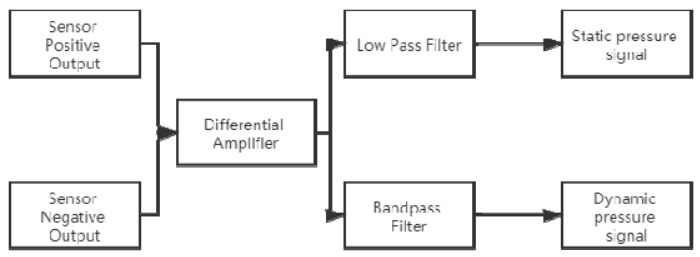

Fig. 3. signal pre-processing module

\subsection{Pressure control module}

The pressure control module is designed to realize the intelligent pressure control of the pulse collector, which including three peristaltic pumps, three slow reducing solenoid valves, three gas storage chambers and silicone tube for conducting gas path. The peristaltic pump uses OEM micro-peristaltic pump, consists of three rotor pump heads and DC motor with $5 \mathrm{~V}$ power supply. The solenoid valve adopts 2p025-06s normally closed solenoid valve with $12 \mathrm{~V}$ power supply and triode.

\subsection{ARM microprocessor module}

Arm microprocessor consists of STM32F373 microcontroller ${ }^{[6]}$ and its associated combined circuitry. After the MCU is powered on, the timer, ADC, DMA, USART and other modules are initialized to complete the initialization parameter setting, and then the handshake flag is sent to the upper computer to establish communication.

\section{Model establishment}

\subsection{The optimal pulse pressure model of the artery}

The blood is regarded as an ideal fluid and described by Euler formula:

$$
\partial \rho / \partial \mathrm{t}+\nabla(\rho v)=0
$$

where $v$ represents speed and t represents time.

In radial artery model, the inflow and outflow volume are equal. The radial artery vascular model is shown in Figure 4, and the column coordinate is used, the $\mathrm{x}$-axis coincides with the tube axis, and $\mathrm{r}$ represents the radial coordinate, $\theta$ is the circular coordinate, the basic equation of blood movement is as follows:

$$
\partial \mathrm{u} / \partial \mathrm{x}+1 / \mathrm{r} *(\partial(\mathrm{rv})) / \partial \mathrm{r}=0
$$

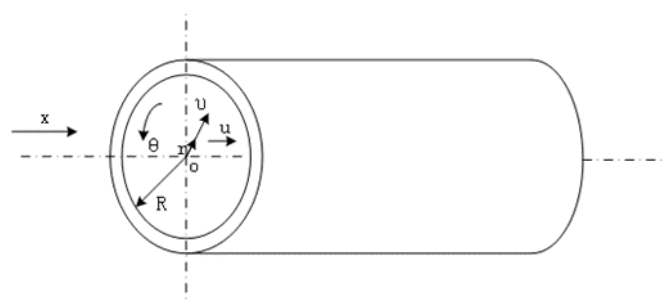

Fig. 4. Radial artery model

In radial artery model, the inflow and outflow volume are equal. The radial artery vascular model is shown in Figure 4, and the column coordinate is used, the $\mathrm{x}$-axis coincides with the tube axis, and $\mathrm{r}$ represents the radial coordinate, $\theta$ is the circular coordinate, the basic equation of blood movement is as follows:

$$
\partial \mathrm{u} / \partial \mathrm{x}+1 / \mathrm{r}(\partial(\mathrm{rv})) / \partial \mathrm{r}=0
$$


Blood vessel wall itself has a certain elasticity and tensility ${ }^{[7]}$. The pulse diagnosis of traditional Chinese medicine (TCM) is to obtain the pulse by pressing the three parts of Cun, Guan and Chi, the patient's information is obtained through the pressure feedback of the fingertip. In this paper, according to the pressure of pulse diagnosis, a mathematical model is established to simulate this process. First of all, the radius of the blood vessels is $\mathrm{r}$, the vascular expansion radius increment is $\Delta r, P$ is the intravascular pressure, that is, the pulse pressure felt by the finger of the doctor, $P e$ is the pressure of the blood vessels, that is, the pressure applied to the artery by the pressure sensor or the Chinese physician's finger. across both columns at the top or the bottom of the page.
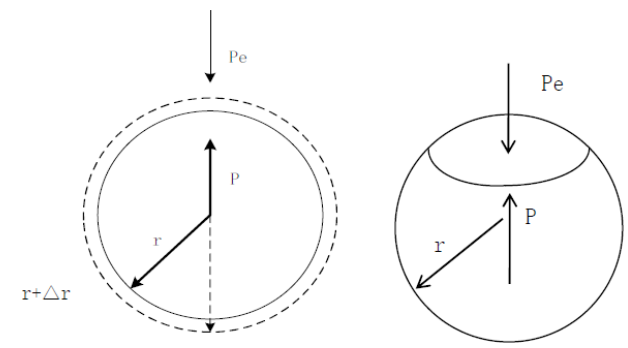

Fig. 5. Pressure model of blood vessel cross section

Figure 5 is a cross-sectional pressure model of blood vessels, let $\Delta p / l$ be the pressure drop on the tube length 1 , $\eta$ is blood viscosity. Poiseuille's Law ${ }^{[8]}$ gives the relationship between volume flow $\mathrm{Q}$ and pressure difference at both ends of the tube $\Delta \mathrm{P}$ when fluid flows in a horizontal circular tube:

$$
Q=\left(\pi R^{\wedge} 4 \Delta p\right) / 8 \eta l
$$

In the actual situation, the blood vessels at the artery are fine, when pressure from sensor or doctor's finger changes, the force area approximation can be regarded as no change, so it can be obtained:

$$
F=P \cdot S-\sigma
$$

$F=\left(8 \mathrm{Q} \eta \mathrm{lE}^{\wedge} 4 \cdot \mathrm{s}\right) /\left(\pi \mathrm{r}^{\wedge} 4\left(\mathrm{E}+\mathrm{P}-\mathrm{P} \_\mathrm{e}\right)^{\wedge} 4\right)-\left(1-\mu^{\wedge} 2\right) \mathrm{r} / \mathrm{h}(\mathrm{E}+\mathrm{P}-$ P_e)(P-P_e )

Discuss the above:

1. When the blood vessel pressure increases from small to large, the value $P-P \_e$ changes from large to small, $\mathrm{F}$ will gradually become larger, when the $\mathrm{P}=\mathrm{P}$ e, F obtains the maximum value $(8 Q \eta l \cdot s) /\left(\pi r^{\wedge} 4\right)$.

2. When $P<P \_e$, the external pressure is larger than the internal pressure, at this time $\left|P-P_{-} e\right|$ is not very large, and the blood vessels remain round under the action of anti-tensile wall of the artery. The value of $\mathrm{F}$ is $(8 Q \eta l \cdot s) /\left(\pi r^{\wedge} 4\right)$.

3. When $P<P \_e$, the external force is still larger than the internal force, but at this time the value $\left|P-P_{-} e\right|$ has exceeded the critical value, the anti-tensile wall of the artery can not withstand external forces, the blood vessels will collapse, so that the cross-sectional area of blood vessels rapidly decreased, resulting in a decrease in blood flow and peripheral resistance increased simultaneously, internal pressure $\mathrm{P}$ and $\mathrm{F}$ also decreased.

\subsection{Applicability analysis of flexible sensor for optimal pulse pressure model}

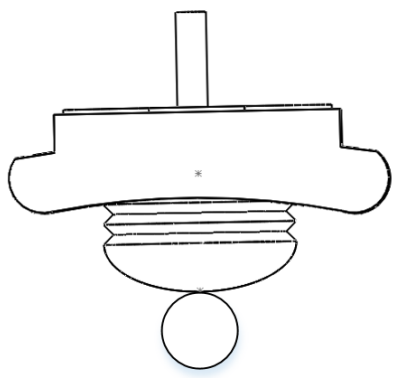

Fig. a.

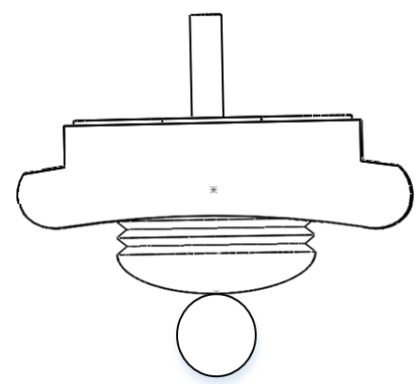

Fig. b.

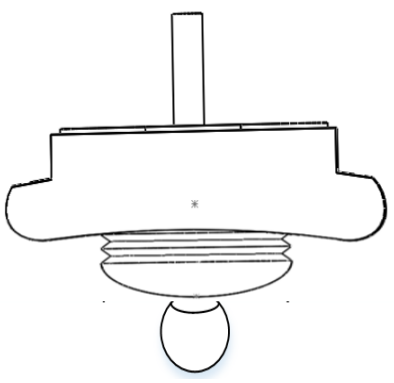

Fig. c.

Fig. 6. Simulation of pulse diagnosis process by flexible sensor

Figure 6 shows the simulation of the pulse diagnosis process by the flexible sensor, figure (a) shows that the flexible sensor has just come into contact with the skin and there is no relative pressure between the contact surfaces. Set at this time the volume of the airbag is $V_{-} 1$, when the pressure between the contact surface gradually increases, the flexible sensor will deform. Figure (b) shows when $P=P_{-} e$, the maximum value of $\mathrm{F}$ is $F=(8 Q \eta l \cdot s) /\left(\pi r^{\wedge} 4\right)$.

The external pressure continues to increase when $P=P \_e$, The pressure difference has exceeded the critical value, anti-tensile wall of the artery can not withstand external forces, blood vessels will collapse, so that the cross-sectional area of blood vessels rapidly decreased, resulting in a decrease in blood flow, peripheral resistance increased simultaneously, internal pressure $\mathrm{P}$ and $\mathrm{F}$ also decreased. Figure (c) shows the simulation of the contact between the flexible sensor and 
the radial artery when the blood vessel collapses. Set the volume of the flexible sensor be $V_{-} 3$.

During the pressurized process, the pressure inside the airbag is related as follows:

$$
P_{-} 1<P_{-} 2<P_{-} 3
$$

Since the sensor and skin just fit to the pressure increasing process, the flexible sensor satisfies the optimal pulse pressure model for the radial artery's signal acquisition process. The pressure in the sensor airbag is increasing, as can be seen from Newton's third law, and the external pressure acting on the radial artery tube is also increasing, so that the whole pressurization process can traverse the pulse pressure range. This model can reflect the law of pulse feedback changing with external pressure, which is consistent with the actual theory of traditional Chinese medicine and can explain the theoretical system more scientifically.
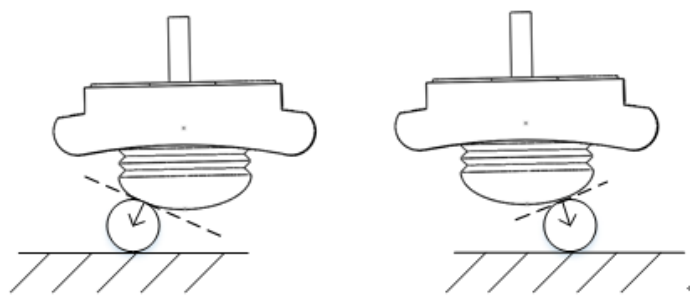

Fig. 7. Non-vertical pressurized model analysis

The short axis of the flexible sensor is $5 \mathrm{~mm}$, and the long axis is $10 \mathrm{~mm}$, which is much larger than the diameter of the radial artery tube. When the radial artery tube is pressed vertically, it meets the above model. As shown in Figure 7, when the pressure position of the flexible sensor deviates to the left or right position of the vascular, the ellipsoid at the lower end of the flexible sensor is always tangent to the vascular, and the vertical pressure condition of the arterial tube is satisfied during the pressure process. Therefore, the use of this model for pulse data collection is more "inclusive" for the selection of pulse location. People who have not received pulse diagnosis knowledge can also collect more accurate pulse information. The use of this collection model can greatly reduce the threshold of users.

\section{Pulse acquisition system performance verification}

In order to verify the accuracy of pulse information collection by the three pulse acquisition systems based on flexible sensors, the data comparison between the pulse collection equipment and the pulse-diagnosis equipment on the market is now made. In the experiment, the data of the pulse acquisition device is compared with the pulse diagnosis device on the market.

\subsection{Acquisition and processing of pulsed signals}

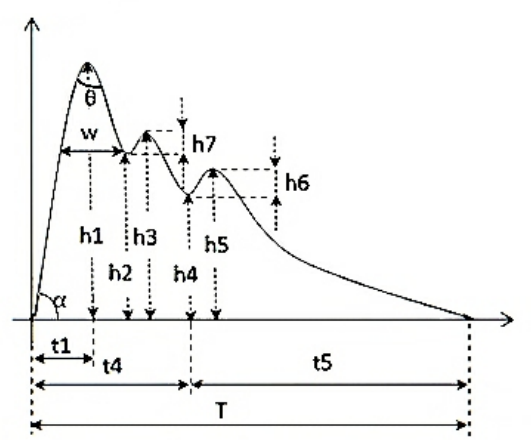

Fig. 8. Characteristic map of pulse data

The subjects selected 30 healthy college students between the ages of 20 and 25 from Tianjin University for data collection, 15 boys and 15 girls each. All subjects were arranged to sit in silence for two minutes before data acquisition, and their arms were placed flat in front of the device, about the height of the heart. The data acquisition point is located in the left hand of "GUAN" position. In the experiment, the equipment is used to obtain pulse information in slow decompression mode, and the upper computer software display is as shown in Figure 9.

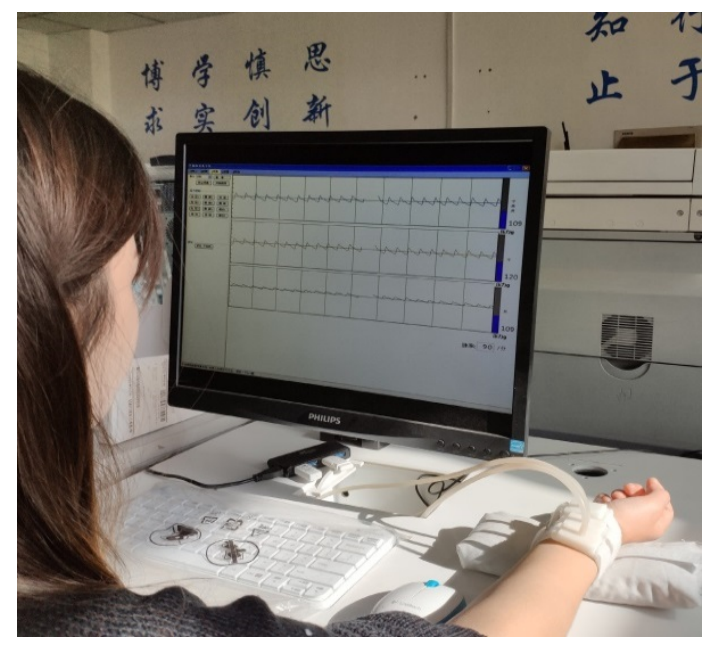

Fig. 9. The upper computer collects the image of pulse information

In order to verify the accuracy of the data collected by this system, the experiment mainly involves the time domain parameters of the pulse data, and the processing of the pulse data by MATLAB tool is mainly to obtain the time domain parameters. In this paper, wavelet transform is used to remove baseline drift and highfrequency noise ${ }^{[9-10]}$, and ILP algorithm is used to extract time-domain characteristics of pulse data ${ }^{[11-12]}$. The signal processed in time domain is shown in figures 10 and 11 . 

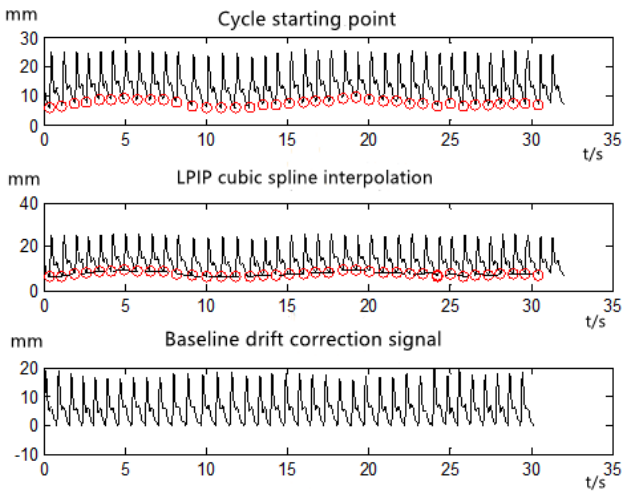

Fig. 10. Pulse signal pre-processing

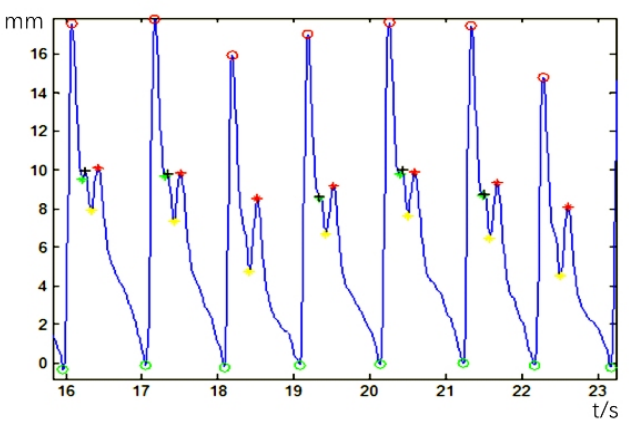

Fig. 11. Time domain feature point marking effect picture

\subsection{Comparison experiment}

In order to verify the accuracy of the pulse information collected by the pulse diagnosis equipment, the data comparison experiment was conducted between the pulse diagnosis equipment and the SMF-III (a standard pulse diagnosis equipment which is mostly used in the hospital). The experimental object is the same as the previous description. In the same period of time, the device is used to collect the pulse data of Cun, Guan and chi. In order to eliminate the interference of other factors, this paper only uses the Guan's data collected by the system to compare with the standard equipment (SMFIII) in the analysis process. The main parameters of comparative analysis are the optimal pulse pressure $\mathrm{F}$ and the time-domain characteristic parameters of physiological significance, such as $\mathrm{H} 1$ (pulse power) and H3 / H1 (pulse shape), and the pulse rate f, which is calculated by taking the reciprocal of cycle $t$.

Ten groups of optimal pulse pressure data were selected from the control data group for statistical analysis. At the same time, the optimal pulse pressure obtained by the two systems was analysed by t-test of double sample equal variance hypothesis, as shown in Table 2 .

Table 1. Ten groups of optimal pulse pressure values were selected.

\begin{tabular}{|c|c|c|c|c|c|}
\hline $\begin{array}{c}\text { NUMB } \\
\text { ER }\end{array}$ & $\begin{array}{c}\text { VARIABL } \\
\text { E1 }\end{array}$ & $\begin{array}{c}\text { VARIABL } \\
\text { E2 }\end{array}$ & $\begin{array}{c}\text { NUMB } \\
\text { ER }\end{array}$ & $\begin{array}{c}\text { VARIABL } \\
\text { E1 }\end{array}$ & $\begin{array}{c}\text { VARIABL } \\
\text { E2 }\end{array}$ \\
\hline 1 & 89 & 87 & 6 & 125 & 117 \\
\hline 2 & 101 & 103 & 7 & 119 & 123 \\
\hline 3 & 135 & 129 & 8 & 88 & 95 \\
\hline 4 & 109 & 110 & 9 & 79 & 83 \\
\hline 5 & 127 & 126 & 10 & 97 & 90 \\
\hline
\end{tabular}

According to the data analysis, there is no obvious difference in the optimal pulse pressure between the two systems, from the following table 3 , the $F$ value is 0.155 , the significance value is 0.698 , which is greater than 0.05 . We cannot refuse the equivariance hypothesis, and the $\mathrm{P}$ value (SIG) is greater than 0.05 . Therefore, there is no significant difference between the optimal pulse pressure obtained by this system and the standard system.

T-test was performed on $\mathrm{H} 1, \mathrm{H} 3 / \mathrm{H} 1$ and $\mathrm{F}$. it can be seen from tables 4,5 and 6 that the $\mathrm{P}$ values (SIG) calculated from $\mathrm{H} 1, \mathrm{H} 3 / \mathrm{H} 1$ and pulse rate $\mathrm{f}$ are all greater than 0.05 . It can be concluded that there is no statistical difference between the pulse data obtained by this system and the standard system in the amplitude parameters, pulse shape, pulse rate and other aspects, which can prove that the data collected by this system is true and reliable.

Table 2. T test results of optimal pulse pressure.

\begin{tabular}{|l|c|c|c|c|c|c|c|}
\hline & $\mathrm{F}$ & $\begin{array}{c}\text { Significance } \\
\text { Level }\end{array}$ & $\mathrm{t}$ & Freedom & Sig. & $\begin{array}{c}\text { Mean } \\
\text { differenc } \\
\mathrm{e}\end{array}$ & $\begin{array}{c}\text { Standard } \\
\text { error } \\
\text { difference }\end{array}$ \\
\hline $\begin{array}{l}\text { Assumed } \\
\text { Equivariance }\end{array}$ & .155 & .698 & .074 & 18 & .942 & .60000 & 8.09046 \\
\hline $\begin{array}{l}\text { Equivariance } \\
\text { is not } \\
\text { assumed }\end{array}$ & & & .074 & 17.796 & .942 & .60000 & 8.09046 \\
\hline
\end{tabular}

Table 3. $\mathrm{T}$ test results of $\mathrm{H} 1$.

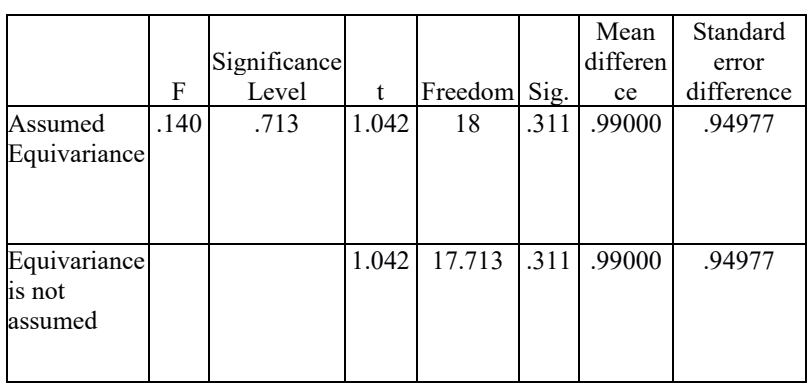

Table 4. T test results of $\mathrm{H} 3$ / $\mathrm{H} 1$.

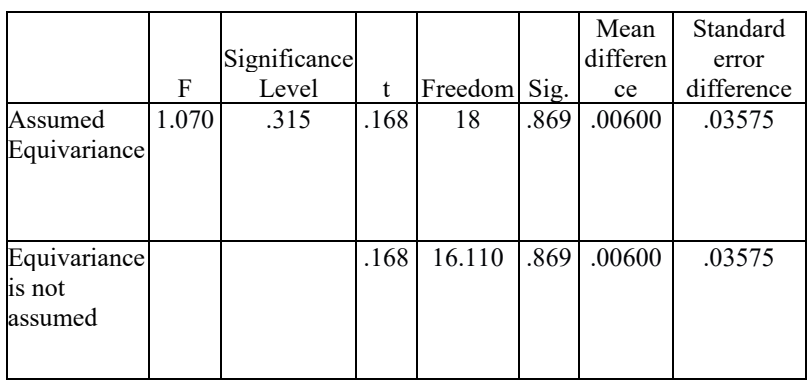

Table 5. $\mathrm{T}$ test results of pulse rate $\mathrm{f}$

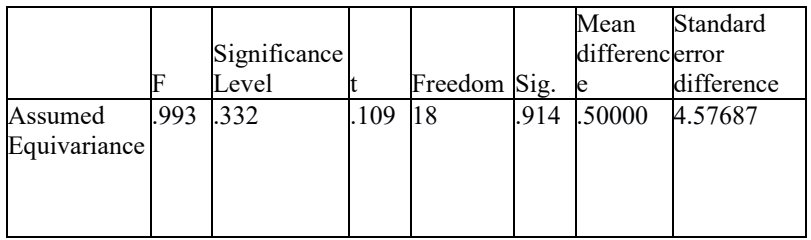




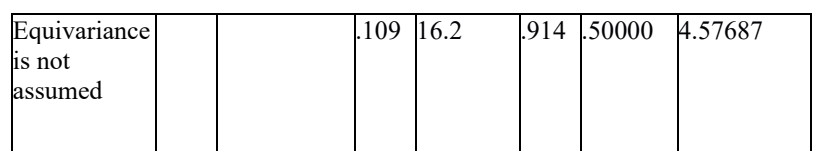

T-test was performed on $\mathrm{H} 1, \mathrm{H} 3 / \mathrm{H} 1$ and $\mathrm{F}$. it can be seen from tables 4,5 and 6 that the $\mathrm{P}$ values (SIG) calculated from $\mathrm{H} 1, \mathrm{H} 3 / \mathrm{H} 1$ and pulse rate $\mathrm{f}$ are all greater than 0.05 . It can be concluded that there is no statistical difference between the pulse data obtained by this system and the standard system in the amplitude parameters, pulse shape, pulse rate and other aspects, which can prove that the data collected by this system is true and reliable.

\section{Conclusions}

By analysing the shortcomings of the existing pulse equipment, this paper designs a new pulse acquisition method, establishes the optimal pulse pressure model, analyses the feasibility of the pulse acquisition equipment based on flexible sensor, and realizes the development of three pulse diagnosis equipment. Through the correlation analysis of the time domain parameters of the data, it shows the real reliability of the data collected by the device, which provides a new way for the current pulse diagnosis acquisition equipment, greatly reduces the loss rate of the pulse diagnosis equipment, improves the user experience, and provides more comprehensive and reliable technical support for the pulse replay technology and telemedicine.

\section{References}

1. Pang Yudan, Liu Jianmin, Zhang libing. Research progress of TCM pulse meter [J]. Beijing Biomedical Engineering, 2014,01:101-107 in Chinses.

2. Tang Weichang, Li Rui. Research on three pulse detection systems [J]. Chinese Journal of medical devices, 2005,03:164-166 in Chinses.

3. Wang Xuemin, Yang Cheng, Lu Xiaozuo, et al. Design of pulse detection system based on flexible array sensor [J]. Journal of sensing technology, 2012,06:733-737 in Chinses.

4. Hong Guang, Gao Dan. Analysis on the development of objectification of pulse diagnosis in traditional Chinese medicine $[\mathrm{J}]$. International Journal of traditional Chinese medicine, 2016,38 (10) ):869-871 in Chinses.

5. LY Wei, CT Lee. New Scientific method of pulse Diagnosis[J]. P Chow - American Journal of Acupuncture, 1984, 12(30): 205-209.

6. Lee HJ, Park YB, Heo U. Implementation of radial pulse diagnosis system using InyoungCheongu comparison method [J]. Journal of Biomedical Engineering Research, 1993,14(1):73-80.

7. M.(Miko)Elwenspoek, RJ Wiegerink. Mechanical Microsensors[M]. Berlin: Springer, $2001.86 \sim 94$.
8. SM Sze. Semiconductor Devices Physics and Technology 2nd Edition[M]. New York City: John Wiley \& Sons, 2001.21-49 .

9. Wang H, Cheng Y. A quantitative system for pulse diagnosis in Traditional.

10. Chinese Medicine.[C]. Conference: International Conference of the IEEE Engineering in Medicine \& Biology Society IEEE Engineering in Medicine \& Biology Society Conference. Conf Proc IEEE Eng Med Biol Soc, 2005:5676-9.

11. Zhang J, Wang R, Lu S, et al. EasiCPRS:design and implementation of a portable Chinese pulse-wave retrieval system: International Conference on Embedded Networked Sensor Systems, SENSYS 2011, Seattle, Wa, Usa, November, 2011[C].

12. $\mathrm{Xu} \mathrm{L}$, Meng $\mathrm{Q} \mathrm{H}$, Wang $\mathrm{K}$, et al. Pulse images recognition using fuzzy neural network[J]. Expert Systems with Applications, 2009,36(2):3805-3811. 